\title{
Oncology Research Output and its Citation Analysis at Continental level: A Study (2003-2012)
}

\author{
Ramesh Pandita ${ }^{1, a}$, Shivendra Singh ${ }^{2, b}$ \\ ${ }^{1}$ BGSB University, R/o 274-C, Durga Nagar Sect. No I, P/o Roop Nagar, \\ Jammu - 180013, Rajouri, Jammu \& Kashmir, India \\ ${ }^{2}$ UCN, BFUHS, Farikdot - 151203, Punjab, India \\ Ph. -+91-1639-250433 \\ a,bE-mail address: rameshpandita90@gmail.com , shiv.mail@gmail.com
}

\begin{abstract}
The present study examines the research output and citation analysis in the field of Oncology, a branch of medical science which deals with the study and treatment of tumours, what we commonly know as cancer. Cancer as a disease is not confined to a particular region or a country, but is a global phenomenon and is still beyond the complete understanding and control of medicos. Research in the field of biomedical sciences in general and oncology is particular is undertaken at global level with almost each country contributing its bit in understating and control of disease. The study makes an empirical assessment of the research output and growth in the field of oncology at continental level for the period 2003-2012 and evaluates the aspects like research growth, citation analysis, h-Index etc. Data for the present study has been retrieved from the SCImago Journal and Country Ranking, which is totally based on the SCOPUS data source. Findings: - A total of 310593 research papers were published across six continents of the world during the period 2003-2012. Europe emerged the largest continent with its publication share of $(124598,40.11 \%)$. Europe is followed by North America with its share percentage of $(102897,33.12 \%)$ and Asia with $(70555,22.71 \%)$. The contribution of Oceania, South America \& Africa to the world oncology research is not that encouraging, as such there is greater need to promote oncology research in these continents. African contribution to global oncology research during the period remained (2215, $0.71 \%)$, South American (3009, $0.96 \%$ ) and Oceania contributed $(7319,2.35 \%)$. Oncology research publication on average during the period of study grew annually at $8.15 \%$, while as at continental level Africa registered highest annual publication growth of $19.08 \%$. North America and Europe are the only continents which recorded publication's growth below the average global growth.
\end{abstract}

Keywords: Oncology Research; Africa; Asia, Europe; North America; Oceania; South America; Research Output; Citation Analysis; H-Index

\section{INTRODUCTION}

Cancer is known as the second largest killer disease after cardiovascular diseases. In 2012 more than 8.2 million [1] deaths were reported across the world due to cancer. The large scale deaths due to cancer can be owed to the fact that medical science has not yet fully developed understanding and control over the disease. Of the late the disease is still 
controllable if detected at the early or initial stage of its occurrence, but the same becomes quite impossible to control if detected in middle or last stage of its occurrence.

Cases of morbidity and mortality by cancer are prevalent in each region of the world. As per The World Cancer Report (2014) more than 32.6 million people across the globe were living with cancer by the end of 2012 [2]. Liver, Lung, Prostate, Colorectal, Stomach is the common organs among males, which suffer with this disease, while as women mostly have been found with suffering from Breast cancer, Colorectal, Lung, Cervix \& Stomach cancers are very common.

Although prevalence of cancer is not confined to any specific region, continent or country, but still the studies so far conducted in this direction have confirmed of high cancer incidence rate with high-income countries, mostly in North America and Western Europe along with Japan, Korea, Australia and New Zealand [3]. As per the World Cancer Report (2014), the disease has made an increasing shift towards the low and middle income countries.

Poor health care and unhygienic life style with increasing population has put these nations at greater risk of suffering with an increase in cancer related incidences. Experts are of the view that due to unhealthy lifestyle annual incidence of cancer in low and middle income countries is likely to grow by $70 \%$ by 2030 [4]. This indeed is the area of concern, whereby low and middle income nations have to take some drastic measure to tackle such kind of outbreak. Need is to put more and more emphasis on the oncology research so that the alarming increase in the cancer incidence cases be put under control to a sustainable level. Africa, Asia \& Central and South America have been detected as high cancer concentration zones with more than $60 \%$ cases of cancer incidence and nearly $70 \%$ cancer related deaths have been reported from these regions [5].

Keeping in view the above fact, the idea to undertake the bibliometric study on the research output in the field of oncology at continental level was conceived. To undertake the preset study data was retrieved on April 29, 2014 from the official website of the SCImago Journal and Country Ranking accessible at:

http://www.scimagojr.com/countryrank.php?area $=2700 \&$ category $=2730 \&$ region $=$ all\&year $=\mathrm{a}$ $11 \&$ order $=$ it\&min $=0 \& \min$ type $=$ it.

The database is purely a SCOPUS data series. The study lasts around all the six continents of the world viz., Africa, Asia, Europe, North America, Oceania and South America.

\section{REVIEW OF LITERATURE}

Oncology is perhaps one of the major biomedical research areas in which a good number of metric studies have already been undertaken by researchers both at the national and global level. Some of the key studies undertaken earlier in the field of oncology and relevant to present study have been reviewed hereunder.

Micheli et al. (2009) [6] undertook a metric study on the cancer research performance in the European Union for the period 2000-2008 and observed that China is one of the fastest emerging research countries in the world in the field of oncology. USA still dominates the global research scene in the field of oncology, while as European Union a conglomerate of 27 nations produced largest oncology publications till 2006. The authors further observed that as per the UNESCO data for the period 2002 to 2007 oncology research expenditure increased by $34 \%$ in North America, $161 \%$ in China \& $28 \%$ in the European Union. 
Grossi, Belvedere \& Rosso (2003) [7] evaluated 3142 publications undertaken in clinical cancer research for the period 1995-1999, covering discussion around chemotherapy combined with other treatments along with impact factor of publications published from each individual country. The authors also draw the comparison of Impact factor between oncology publications between the European Union and North America in which North American publication emerged better over the EU. United States with $37.7 \%$ publication share percentage emerged the leading oncology research country, followed by Italy, United Kingdom and Japan.

Hortobagyi et al. (2005) [8] undertook study over the breast cancer incidence, mortality and survival rate across different regions of the world which authors found varying considerably from country to country and region to region. The authors underlie various complex factors like population structure, lifestyle, environment and socioeconomic status that play a part in the incidence, mortality and survival of breast cancer. Glynn et al. (2010) ${ }^{[9]}$ undertook a bibliometric study on breast cancer research output for the period 1945-2008 by analysing 180126 publications concerning breast cancer and found that the United States as the largest contributor to the breast cancer research followed by the United Kingdom and Germany. The researchers further found that there is a growing trend towards collaborative research among nations and united stated again was found the leading nation in this sphere. Furthermore, the researchers revealed that collaborative publications are the ones which are more cited.

Ugolini and Mela (2003) [10] evaluated 66021 research papers in oncology published from 1996-2000 and found that 35.5\% came from the European Union, while as maximum $38.8 \%$ came alone from the US. While drawing comparison with earlier oncology bibliometric studies the authors found that the top five oncology research countries of the European Union maintained their ranking while as smaller countries like Denmark, Norway and Ireland feared worse in 2000 and the nations which showed improvements where France, Germany and Greece.

Glynn et al. (2010) [11] examined the representation of cancer in the medical literature by evaluating 63260 entries from PubMed and 126845 entries from WoS and found that 26 neoplasms accounted for $25 \%$ of the total biomedical research output with cancers like breast, Prostate, Lung, Intestinal cancer and Leukaemia dominating the research output in top oncology research journals. The Authors further observed that there is a disproportionate representation of oncology research in the leading biomedical journals.

Ortiz et al. (2009) [12] undertook bibliometric study of cancer research in Puerto Rico for the period 1903-2005 by evaluating 369 articles and found that majority $39.6 \%$ publications were university publications, $72.1 \%$ articles were written in English language, $69.6 \%$ articles were original research papers and the studies were mostly concerned with digestive cancer studies and Gynecology. The authors further observed that although research in the field of oncology has increased significantly in the country, especially post 1913 but the rate of cancer mortality has increased even at a much faster rate.

\section{PROBLEM STATEMENT}

Research is the backbone of every subject field. Research not just helps a subject entity to survive and sustain under the prevailing circumstances, but also helps it to lay down a path for its furtherance. All the sciences are bound to suffer if they fail to add new knowledge to their existing pool of knowledge. Pure \& applied sciences are more susceptible to obsolesce at a greater pace than the social sciences, hence there is a greater degree of need to undertake 
constant and continuous research in these very areas. Biomedical science is one such important area where scientific investigations become obsolete at a greater pace, hence constant and continuous research is the only way out which helps biomedical sciences to keep pace with the requirements of the robust health sector.

\section{OBJECTIVES OF THE STUDY}

The study aims to evaluate the research output in the field of oncology at continental level along with other allied areas, undertaken during the last decade viz., for the period 2003-2012. To assess the annual research growth in the given field at both continental and global level, along with aspects like citation analysis and h-index, which more or less have become the parameters to assess the quality of research in any given filed.

\section{METHODOLOGY \& APPROACH}

Present study has been purely undertaken on secondary data, retrieved from official website of SCImago Journal and Country Ranking on April 29, 2014 accessible at Http://www.scimagojr.com/countryrank.php?area=2700\&category=2730\&region=all\&year= all\&order $=i$ it\&min $=0 \&$ min type $=i t$. Given the objects of the study, the data upon retrieval was in a semi structured form, hence was structured by segregating countries as per their affiliation to different continents of the world, and to do the needful, world atlas were used, accessible at www.worldatlas.com. To evaluate the data various statistical \& mathematical tools and techniques were employed. The study leaves enough scope to study the allied aspects of oncology research both at regional and country level.

\section{DISCUSSION \& DATA ANALYAIS}

Table I . Distribution of oncology documents produced at continental level.

\begin{tabular}{|c|c|c|c|c|c|c|c|}
\hline $\begin{array}{l}\text { Continent } \rightarrow \\
\quad \text { Year } \downarrow\end{array}$ & $\begin{array}{c}\text { Africa } \\
\text { NP (CG\%) }\end{array}$ & $\begin{array}{c}\text { Asia } \\
\text { NP (CG\%) }\end{array}$ & $\begin{array}{c}\text { Europe } \\
\text { NP }(C G \%)\end{array}$ & $\begin{array}{l}\text { N.America } \\
\text { NP (CG\%) }\end{array}$ & $\begin{array}{c}\text { Oceania } \\
\text { NP (CG\%) }\end{array}$ & $\begin{array}{l}\text { S.America } \\
\text { NP (CG\%) }\end{array}$ & $\begin{array}{c}\text { World } \\
\text { NP (Share\%)* } \\
\&(\text { CG\%) }\end{array}$ \\
\hline 2003 & $\begin{array}{l}94 \\
(-)\end{array}$ & $\begin{array}{c}3412 \\
(-)\end{array}$ & $\begin{array}{c}8958 \\
(-)\end{array}$ & $\begin{array}{c}7074 \\
(-)\end{array}$ & $\begin{array}{c}373 \\
(-)\end{array}$ & $\begin{array}{c}154 \\
(-)\end{array}$ & $\begin{array}{c}20065(6.46)^{*} \\
(-)\end{array}$ \\
\hline 2004 & $\begin{array}{c}96 \\
(2.12)\end{array}$ & $\begin{array}{c}3576 \\
(4.80)\end{array}$ & $\begin{array}{l}9474 \\
(5.76)\end{array}$ & $\begin{array}{c}7974 \\
(12.72)\end{array}$ & $\begin{array}{c}453 \\
(21.44)\end{array}$ & $\begin{array}{c}156 \\
(1.29)\end{array}$ & $\begin{array}{c}21729(6.99)^{*} \\
(8.29)\end{array}$ \\
\hline 2005 & $\begin{array}{c}104 \\
(8.33)\end{array}$ & $\begin{array}{c}4495 \\
(25.69) \\
\end{array}$ & $\begin{array}{c}10642 \\
(12.32) \\
\end{array}$ & $\begin{array}{c}9062 \\
(13.64) \\
\end{array}$ & $\begin{array}{c}521 \\
(15.01) \\
\end{array}$ & $\begin{array}{c}160 \\
(2.56)\end{array}$ & $\begin{array}{c}24984(8.04)^{*} \\
(14.97)\end{array}$ \\
\hline 2006 & $\begin{array}{c}155 \\
(49.03) \\
\end{array}$ & $\begin{array}{c}5406 \\
(20.26)\end{array}$ & $\begin{array}{c}11949 \\
(12.28) \\
\end{array}$ & $\begin{array}{c}10051 \\
(10.91)\end{array}$ & $\begin{array}{c}643 \\
(23.41) \\
\end{array}$ & $\begin{array}{c}261 \\
(63.12) \\
\end{array}$ & $\begin{array}{c}28465(9.16)^{*} \\
(13.93)\end{array}$ \\
\hline 2007 & $\begin{array}{c}137 \\
(-11.61)\end{array}$ & $\begin{array}{c}6142 \\
(13.61)\end{array}$ & $\begin{array}{l}12057 \\
(0.90)\end{array}$ & $\begin{array}{l}10299 \\
(2.46)\end{array}$ & $\begin{array}{c}598 \\
(-6.99)\end{array}$ & $\begin{array}{c}253 \\
(-3.06)\end{array}$ & $\begin{array}{c}29486(9.49)^{*} \\
(3.58)\end{array}$ \\
\hline 2008 & $\begin{array}{c}234 \\
(70.80)\end{array}$ & $\begin{array}{c}7282 \\
(18.56)\end{array}$ & $\begin{array}{l}12984 \\
(7.68)\end{array}$ & $\begin{array}{l}10602 \\
(2.94)\end{array}$ & $\begin{array}{c}847 \\
(41.63)\end{array}$ & $\begin{array}{c}320 \\
(26.48)\end{array}$ & $\begin{array}{c}32269(10.38)^{*} \\
(9.43)\end{array}$ \\
\hline 2009 & $\begin{array}{c}251 \\
(7.26)\end{array}$ & $\begin{array}{c}8309 \\
(14.10)\end{array}$ & $\begin{array}{l}13608 \\
(4.80)\end{array}$ & $\begin{array}{l}11056 \\
(4.28)\end{array}$ & $\begin{array}{c}836 \\
(-1.29)\end{array}$ & $\begin{array}{c}369 \\
(15.31)\end{array}$ & $\begin{array}{c}34429(11.08)^{*} \\
(6.69)\end{array}$ \\
\hline 2010 & $\begin{array}{c}353 \\
(40.63)\end{array}$ & $\begin{array}{c}9426 \\
(13.44)\end{array}$ & $\begin{array}{l}13744 \\
(0.99)\end{array}$ & $\begin{array}{l}11489 \\
(3.91)\end{array}$ & $\begin{array}{c}894 \\
(6.93)\end{array}$ & $\begin{array}{c}336 \\
(-8.94)\end{array}$ & $\begin{array}{c}36242(11.66)^{*} \\
(5.26)\end{array}$ \\
\hline
\end{tabular}




\begin{tabular}{|c|c|c|c|c|c|c|c|}
\hline 2011 & $\begin{array}{c}354 \\
(0.28) \\
\end{array}$ & $\begin{array}{c}10372 \\
(10.03) \\
\end{array}$ & $\begin{array}{l}14954 \\
(8.80) \\
\end{array}$ & $\begin{array}{l}12147 \\
(5.72) \\
\end{array}$ & $\begin{array}{c}1040 \\
(16.33) \\
\end{array}$ & $\begin{array}{c}444 \\
(32.14) \\
\end{array}$ & $\begin{array}{c}39311(12.65)^{*} \\
(8.46)\end{array}$ \\
\hline 2012 & $\begin{array}{c}437 \\
(23.44)\end{array}$ & $\begin{array}{c}12135 \\
(16.99)\end{array}$ & $\begin{array}{l}16228 \\
(8.51)\end{array}$ & $\begin{array}{l}13143 \\
(8.19)\end{array}$ & $\begin{array}{l}1114 \\
(7.11)\end{array}$ & $\begin{array}{c}556 \\
(25.22)\end{array}$ & $\begin{array}{c}43613(14.04)^{*} \\
(10.94)\end{array}$ \\
\hline $\begin{array}{c}\text { Total } \\
(\text { Share\%)\% }\end{array}$ & $\begin{array}{c}2215(0.71)^{*} \\
\quad(19.08)\end{array}$ & $\begin{array}{c}70555 \\
(22.71)^{*} \\
(13.74)\end{array}$ & $\begin{array}{c}124598 \\
(40.11)^{*} \\
(6.20)\end{array}$ & $\begin{array}{c}102897 \\
(33.12)^{*} \\
(6.47)\end{array}$ & $\begin{array}{c}7319 \\
(2.35)^{*} \\
(12.35)\end{array}$ & $\begin{array}{c}3009 \\
(0.96)^{*} \\
(15.41)\end{array}$ & $\begin{array}{c}310593(100)^{*} \\
(8.15)\end{array}$ \\
\hline
\end{tabular}

NP - Number of Publications, CG \% - Corresponding Growth Percentage

Expressions like percentage etc. at all the places has been drawn up to two decimal places and has not been rounded off, so at places may reflect a slight variation while computing data for $100 \%$ figure.

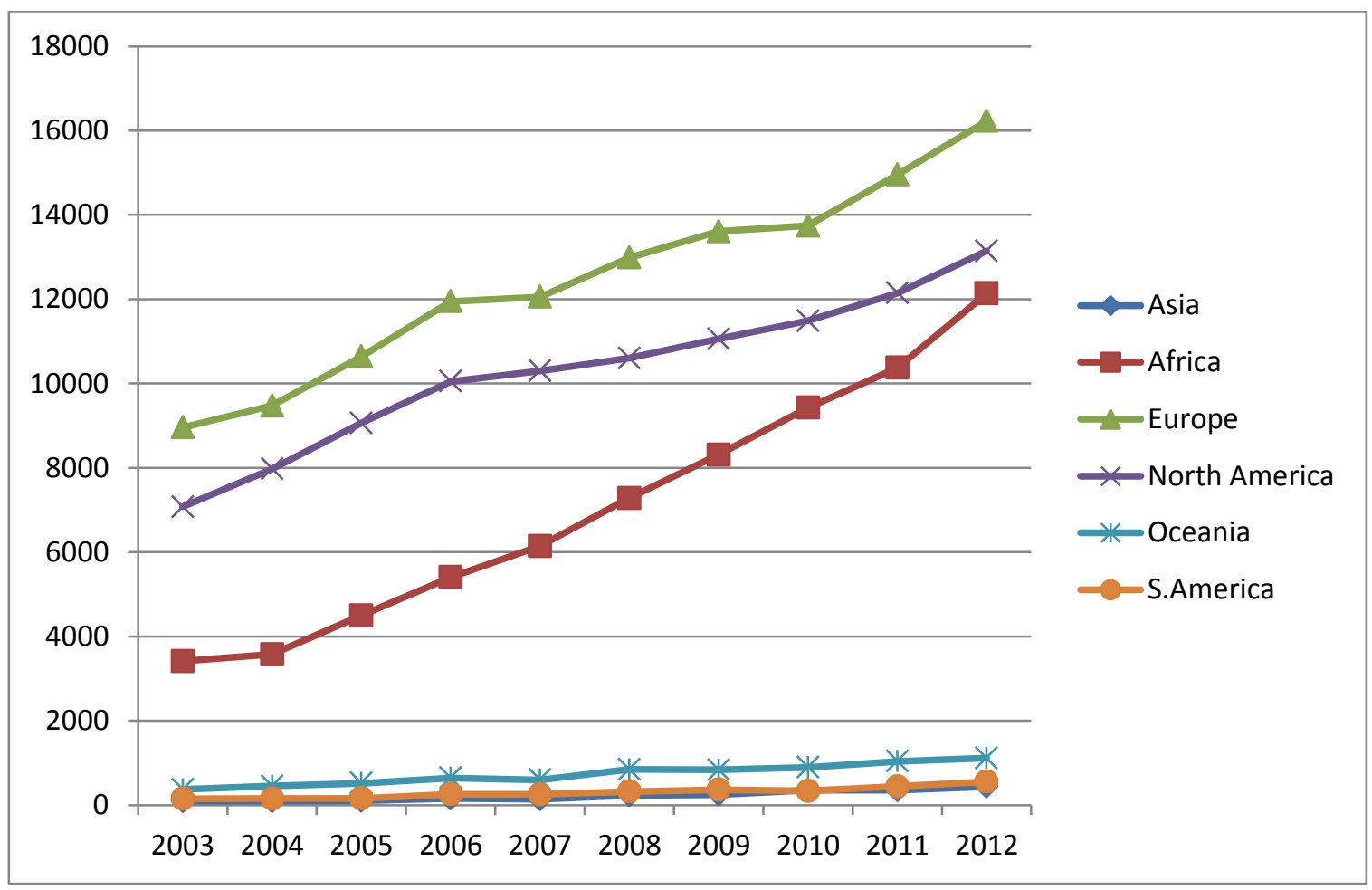

Figure 1. Continental Growth, Distribution Curves of oncology publications.

Above tabulation reflects the growth and distribution of oncology publications during the period of study across different continents of the world. A total of 310593 research publications were published in the field of oncology at global level during the period of study with a maximum $(43613,14.04 \%)$ publications were published during the year 2012. Europe emerged the largest contributor of oncology research with a continental share in $(124598$, $40.11 \%)$, followed by North America \& Asia with share percentage of $(102897,33.12 \%) \&$ $(70555,22.71 \%)$ respectively. Africa and South America are the two continents whose contribution to the oncology research during the period of the study remained below $1 \%$ viz. $(2215,0.71 \%) \&(3009,0.96 \%)$. The contribution of Oceania is equally abysmal, when compared to leading three continents which a share percentage of meager $(7319,2.35 \%)$ 
Annual growth of oncology publications at global level for the period remained $8.15 \%$, while as at continental level Africa emerged the leading continent with an annual publication's growth of $19.08 \%$, followed by South America \& Oceania with an annual growth of $15.41 \% \& 12.35 \%$ respectively. The reason for the high growth percentage of lesser contributing continents is for the fact that even a small increase in the corresponding growth by these continents is going to make a bigger difference in their annual growth, while as the same is not the case with continents conducting research at large scale in the given field, as this will reflect a proportionate increase, hence may not make much difference to their existing growth. Annual growth of publication in Asia was recorded at $13.74 \%$, North America, $6.47 \%$ and Europe $6.20 \%$.

Table II. Distribution of Citable Documents.

\begin{tabular}{|c|c|c|c|c|c|c|c|}
\hline $\begin{array}{l}\text { Continent } \rightarrow \\
\text { Year } \downarrow\end{array}$ & $\begin{array}{c}\text { Africa } \\
\text { NP }(\text { CG\%) }\end{array}$ & $\begin{array}{c}\text { Asia } \\
\text { NP }(C G \%)\end{array}$ & $\begin{array}{c}\text { Europe } \\
\text { NP }(C G \%)\end{array}$ & $\begin{array}{c}\text { N.America } \\
\text { NP } \\
(\mathrm{CG} \%)\end{array}$ & $\begin{array}{c}\text { Oceania } \\
\text { NP }(C G \%)\end{array}$ & $\begin{array}{c}\text { S.America } \\
\text { NP } \\
(\mathrm{CG} \%)\end{array}$ & $\begin{array}{c}\text { World } \\
\text { NP (Share\%) } \% \\
\text { \& }(\text { CG\%) }\end{array}$ \\
\hline 2003 & $\begin{array}{l}89 \\
(-)\end{array}$ & $\begin{array}{c}3309 \\
(-)\end{array}$ & $\begin{array}{c}8301 \\
(-)\end{array}$ & $\begin{array}{c}6480 \\
(-)\end{array}$ & $\begin{array}{c}343 \\
(-)\end{array}$ & $\begin{array}{c}141 \\
(-)\end{array}$ & $\begin{array}{c}18663(6.63)^{*} \\
(-)\end{array}$ \\
\hline 2004 & $\begin{array}{c}90 \\
(1.12) \\
\end{array}$ & $\begin{array}{c}3421 \\
(3.38) \\
\end{array}$ & $\begin{array}{c}8693 \\
(4.72) \\
\end{array}$ & $\begin{array}{c}7156 \\
(10.43) \\
\end{array}$ & $\begin{array}{c}416 \\
(21.28) \\
\end{array}$ & $\begin{array}{c}146 \\
(3.54) \\
\end{array}$ & $\begin{array}{c}19922(7.07)^{*} \\
(6.74)\end{array}$ \\
\hline 2005 & $\begin{array}{c}100 \\
(11.11)\end{array}$ & $\begin{array}{c}4266 \\
(24.70)\end{array}$ & $\begin{array}{c}9613 \\
(10.58)\end{array}$ & $\begin{array}{l}7860 \\
(9.83)\end{array}$ & $\begin{array}{c}468 \\
(12.50)\end{array}$ & $\begin{array}{c}141 \\
(-3.42)\end{array}$ & $\begin{array}{c}22448(7.79)^{*} \\
(12.67)\end{array}$ \\
\hline 2006 & $\begin{array}{c}153 \\
(53.00) \\
\end{array}$ & $\begin{array}{c}5172 \\
(21.23) \\
\end{array}$ & $\begin{array}{c}10910 \\
(13.49) \\
\end{array}$ & $\begin{array}{c}8887 \\
(13.06) \\
\end{array}$ & $\begin{array}{c}573 \\
(22.43) \\
\end{array}$ & $\begin{array}{c}241 \\
(70.92) \\
\end{array}$ & $\begin{array}{c}25936(9.21)^{*} \\
(15.53)\end{array}$ \\
\hline 2007 & $\begin{array}{c}134 \\
(-12.41) \\
\end{array}$ & $\begin{array}{c}5900 \\
(14.07) \\
\end{array}$ & $\begin{array}{l}10959 \\
(0.44) \\
\end{array}$ & $\begin{array}{r}9125 \\
(2.67) \\
\end{array}$ & $\begin{array}{c}545 \\
(-4.88) \\
\end{array}$ & $\begin{array}{c}232 \\
(-3.73) \\
\end{array}$ & $\begin{array}{c}26895(9.55)^{*} \\
(3.69)\end{array}$ \\
\hline 2008 & $\begin{array}{c}200 \\
(49.25)\end{array}$ & $\begin{array}{c}6992 \\
(18.50)\end{array}$ & $\begin{array}{l}11676 \\
(6.54)\end{array}$ & $\begin{array}{l}9238 \\
(1.23)\end{array}$ & $\begin{array}{c}755 \\
(38.53) \\
\end{array}$ & $\begin{array}{c}293 \\
(26.29)\end{array}$ & $\begin{array}{c}29154(10.35)^{*} \\
(8.39)\end{array}$ \\
\hline 2009 & $\begin{array}{c}222 \\
(11.00)\end{array}$ & $\begin{array}{c}7914 \\
(13.18) \\
\end{array}$ & $\begin{array}{l}12282 \\
(5.19) \\
\end{array}$ & $\begin{array}{c}9620 \\
(4.13) \\
\end{array}$ & $\begin{array}{c}751 \\
(-0.52) \\
\end{array}$ & $\begin{array}{c}339 \\
(15.69) \\
\end{array}$ & $\begin{array}{c}31128(11.05)^{*} \\
(6.77)\end{array}$ \\
\hline 2010 & $\begin{array}{c}314 \\
(41.44) \\
\end{array}$ & $\begin{array}{c}8883 \\
(12.24) \\
\end{array}$ & $\begin{array}{l}12362 \\
(0.65) \\
\end{array}$ & $\begin{array}{l}10106 \\
(5.05) \\
\end{array}$ & $\begin{array}{c}799 \\
(6.39) \\
\end{array}$ & $\begin{array}{c}313 \\
(-7.66) \\
\end{array}$ & $\begin{array}{c}32777(11.64)^{*} \\
(5.29)\end{array}$ \\
\hline 2011 & $\begin{array}{c}324 \\
(3.18)\end{array}$ & $\begin{array}{l}9584 \\
(7.89)\end{array}$ & $\begin{array}{l}13478 \\
(9.02)\end{array}$ & $\begin{array}{l}10776 \\
(6.62)\end{array}$ & $\begin{array}{c}930 \\
(16.39)\end{array}$ & $\begin{array}{c}390 \\
(24.60)\end{array}$ & $\begin{array}{c}35482(12.60)^{*} \\
(8.25)\end{array}$ \\
\hline 2012 & $\begin{array}{c}407 \\
(25.61)\end{array}$ & $\begin{array}{c}11136 \\
(16.19)\end{array}$ & $\begin{array}{l}14578 \\
(8.16)\end{array}$ & $\begin{array}{l}11462 \\
(6.36)\end{array}$ & $\begin{array}{l}1001 \\
(7.63)\end{array}$ & $\begin{array}{c}484 \\
(24.10)\end{array}$ & $\begin{array}{c}39068(13.87)^{*} \\
(10.10)\end{array}$ \\
\hline $\begin{array}{c}\text { Total } \\
\text { (Share\%)* } \\
(\text { Avg } \\
\text { CG\%) } \rightarrow\end{array}$ & $\begin{array}{c}2033 \\
(0.72)^{*} \\
(18.33)\end{array}$ & $\begin{array}{c}66577 \\
(23.65)^{*} \\
(13.13)\end{array}$ & $\begin{array}{c}112852 \\
(40.09)^{*} \\
(5.87)\end{array}$ & $\begin{array}{c}907100 \\
(32.22)^{*} \\
(5.93)\end{array}$ & $\begin{array}{c}6581 \\
(2.33)^{*} \\
(11.97)\end{array}$ & $\begin{array}{c}2720 \\
(0.96)^{*} \\
(15.03)\end{array}$ & $\begin{array}{c}281473(100) \\
\quad(7.74)\end{array}$ \\
\hline
\end{tabular}

NP - Number of Publications, CG \% - Corresponding Growth Percentage

Citable documents are generally seen differently from those documents which may not or cannot be cited for different reasons. Given the fact, above tabulation analysis, distribution of citable oncology publications for different continents. At the global level a total of 281473 suitable publications was produced during the period of study, constituting $90.62 \%$ of the total oncology publications, with a maximum $(39068,13.87 \%$ ) published during the year 2012. The scenario in this tabulation is almost similar to that of the table-I with maximum $40.09 \%$ citable documents published from Europe, followed by North America and Asia with a share percentage of $32.22 \% \& 23.65 \%$ respectively. Africa and South America has a less than $1 \%$ contribution and Oceania $2.33 \%$. 


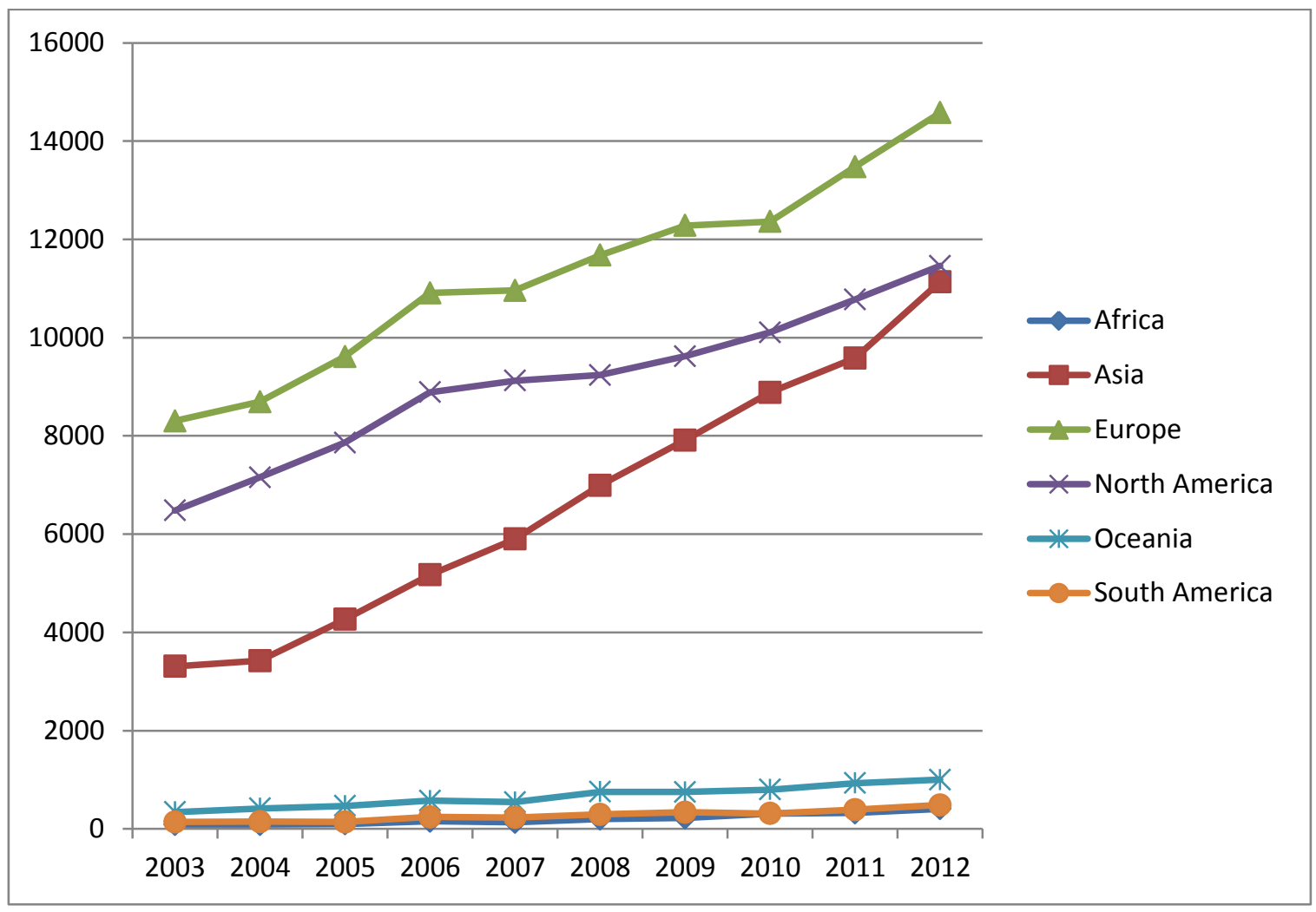

Figure 2. Continental Growth Distribution of Citable Documents.

Table III. Citation Received By Oncology Publication during the Period of Study.

\begin{tabular}{|c|c|c|c|c|c|c|c|}
\hline $\begin{array}{c}\text { Continent } \rightarrow \\
\text { Year } \downarrow\end{array}$ & Africa & Asia & Europe & N. America & Oceania & S. America & $\begin{array}{c}\text { World } \\
\text { NP (Share \%) }\end{array}$ \\
\hline $\mathbf{2 0 0 3}$ & 3075 & 95458 & 311925 & 307507 & 14439 & 4635 & $737039(14.04)$ \\
\hline $\mathbf{2 0 0 4}$ & 3239 & 97051 & 301245 & 309571 & 17576 & 5554 & $734236(13.98)$ \\
\hline $\mathbf{2 0 0 5}$ & 1418 & 96614 & 330259 & 317418 & 17828 & 4116 & $767653(14.62)$ \\
\hline $\mathbf{2 0 0 6}$ & 3169 & 99960 & 312143 & 305360 & 17021 & 7850 & $745503(14.20)$ \\
\hline $\mathbf{2 0 0 7}$ & 1388 & 86753 & 259923 & 267063 & 14083 & 4615 & $633825(12.07)$ \\
\hline $\mathbf{2 0 0 8}$ & 3042 & 88603 & 237615 & 224840 & 16259 & 4457 & $574816(10.94)$ \\
\hline $\mathbf{2 0 0 9}$ & 3011 & 75673 & 215349 & 181951 & 11907 & 4141 & $491032(9.35)$ \\
\hline $\mathbf{2 0 1 0}$ & 2215 & 56292 & 142852 & 122797 & 8515 & 3704 & $336375(6.40)$ \\
\hline $\mathbf{2 0 1 1}$ & 768 & 29518 & 81971 & 65629 & 5554 & 1582 & $185022(3.52)$ \\
\hline $\mathbf{2 0 1 2}$ & 204 & 7465 & 19240 & 15202 & 1313 & 586 & $44010(0.83)$ \\
\hline Total & 20529 & 733387 & 2212522 & 2117338 & 124495 & 41240 & 5249511 \\
(Share\%) & $(0.39)$ & $(13.97)$ & $(42.14)$ & $(40.33)$ & $(2.37)$ & $(0.78)$ & $(100)$ \\
\hline
\end{tabular}

Annual growth of citable documents at global level remained at $7.74 \%$, while as Africa tops the list with an annual growth of $18.33 \%$, followed by South America \& Oceania with an average annual growth of $15.03 \%$ \& $11.97 \%$. During the period of study, Asia observed an annual growth of $13.13 \%$, North America $5.93 \%$ and Europe $5.87 \%$.

At the global level during the period of study, a total of 5249511 citations was received by 310593 documents published during the same period, which on average constitutes 16.90 
citations per document. From each corresponding year if on one hand there is an increase in the number of publications on the other hand, there is a decrease in the citations received for obvious reasons. But after a certain period this trend also assumes a sort of saturation, especially if we look at the cited figures beyond a decade or two. This gets better corroborated by the fact that a maximum $(767653,14.62 \%)$ citations share at the global level was recorded during the year 2005.

In terms of citations share percentage, Europe leads the table with a share percentage of (2212522, $42.14 \%)$, followed by North America \& Asia with a share percentage of $(2117338,40.33 \%) \&(733387,13.87 \%)$. Africa \& South America has less than $1 \%$ citation share, while as Oceania recorded $(124495,2.37 \%)$ share.

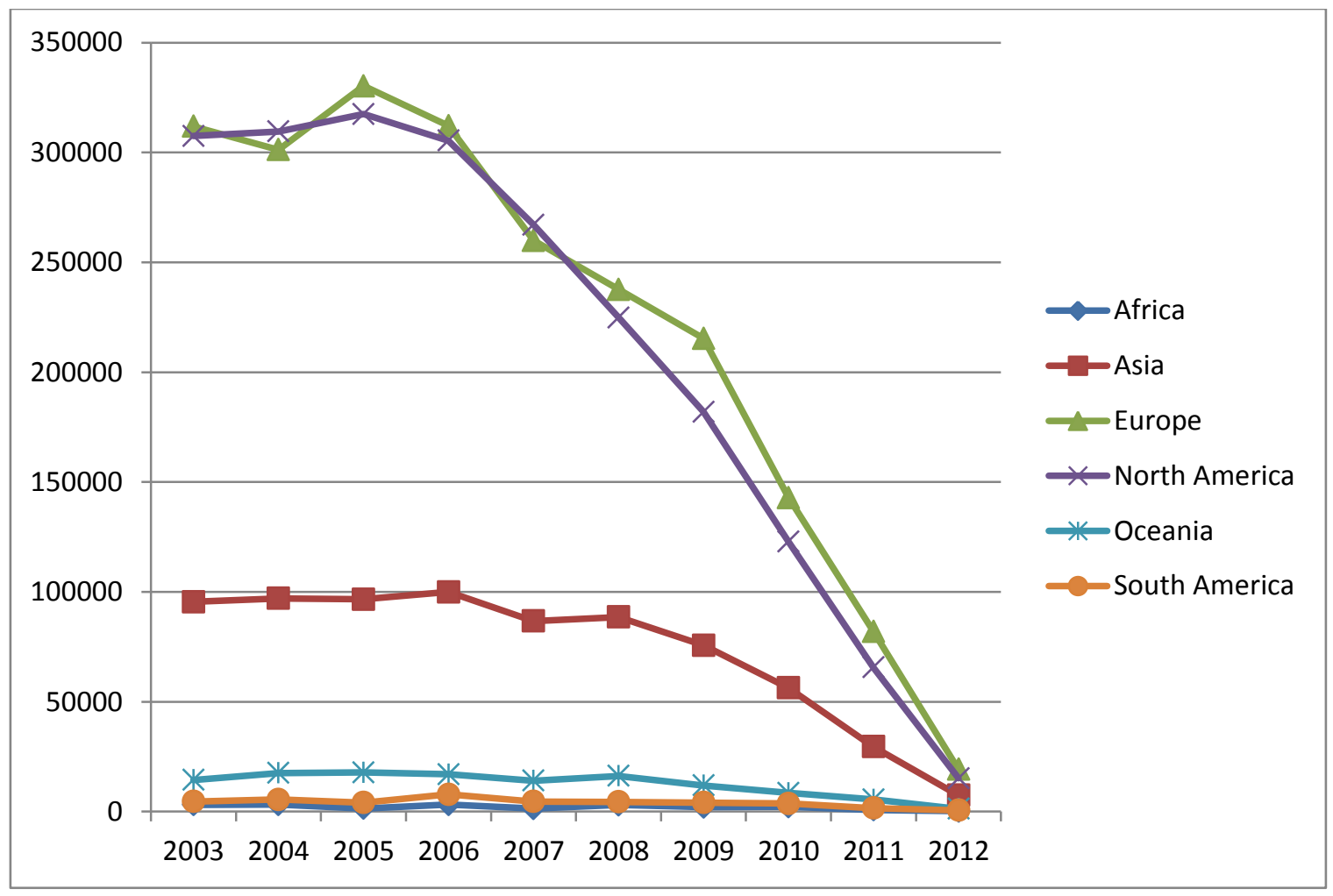

Figure 3. Citation curves of oncology publications at continental level.

In terms of average citations received by each research publication of each continent given the total citations received by each continent respectively, North America leads the table with an average of 20.57 citations per document, followed by Europe \& Oceania with an average of $17.75 \& 17.00$ citations per document. South America 13.70 citations per document, Asia $10.39 \&$ Africa on average received 9.26 citations per documents. This is also somewhere an indicator of the fact that oncology publication from Europe and North America are more popular among the oncology researchers across the globe. Though receiving a higher or greater number of citations cannot be argued about the publication being of quality research, but this somewhere has become a parameter to assess the worthiness of a publication. 
Table IV. Self Citations Received By Oncology Publication during the Period of Study.

\begin{tabular}{|c|c|c|c|c|c|c|c|}
\hline $\begin{array}{c}\text { Continent } \rightarrow \\
\text { Year } \downarrow\end{array}$ & Africa & Asia & Europe & N. America & Oceania & S. America & $\begin{array}{c}\text { World } \\
\text { (Share\%) }\end{array}$ \\
\hline $\mathbf{2 0 0 3}$ & 263 & 20725 & 47275 & 139480 & 1875 & 496 & $210114(13.98)$ \\
\hline $\mathbf{2 0 0 4}$ & 170 & 21450 & 46292 & 138843 & 2142 & 661 & $209558(13.94)$ \\
\hline $\mathbf{2 0 0 5}$ & 212 & 21222 & 50341 & 141306 & 2234 & 519 & $215834(14.36)$ \\
\hline $\mathbf{2 0 0 6}$ & 221 & 22010 & 48557 & 138882 & 2419 & 660 & $212749(14.15)$ \\
\hline $\mathbf{2 0 0 7}$ & 177 & 20050 & 44043 & 120654 & 1947 & 642 & $187513(12.47)$ \\
\hline $\mathbf{2 0 0 8}$ & 313 & 21342 & 39805 & 101418 & 2352 & 677 & $165907(11.03)$ \\
\hline $\mathbf{2 0 0 9}$ & 210 & 18724 & 35987 & 81774 & 1675 & 605 & $138975(9.24)$ \\
\hline $\mathbf{2 0 1 0}$ & 222 & 14123 & 24352 & 55570 & 1251 & 296 & $95814(6.37)$ \\
\hline $\mathbf{2 0 1 1}$ & 77 & 7743 & 14341 & 30281 & 792 & 183 & $53417(3.55)$ \\
\hline $\mathbf{2 0 1 2}$ & 35 & 1861 & 3574 & 7250 & 246 & 53 & $13019(0.86)$ \\
\hline Total & 1900 & 169250 & 354567 & 955458 & 16933 & 4792 & 1502900 \\
(Share\%) & $(0.12)$ & $(11.26)$ & $(23.59)$ & $(63.57)$ & $(1.12)$ & $(0.31)$ & $(100)$ \\
\hline
\end{tabular}

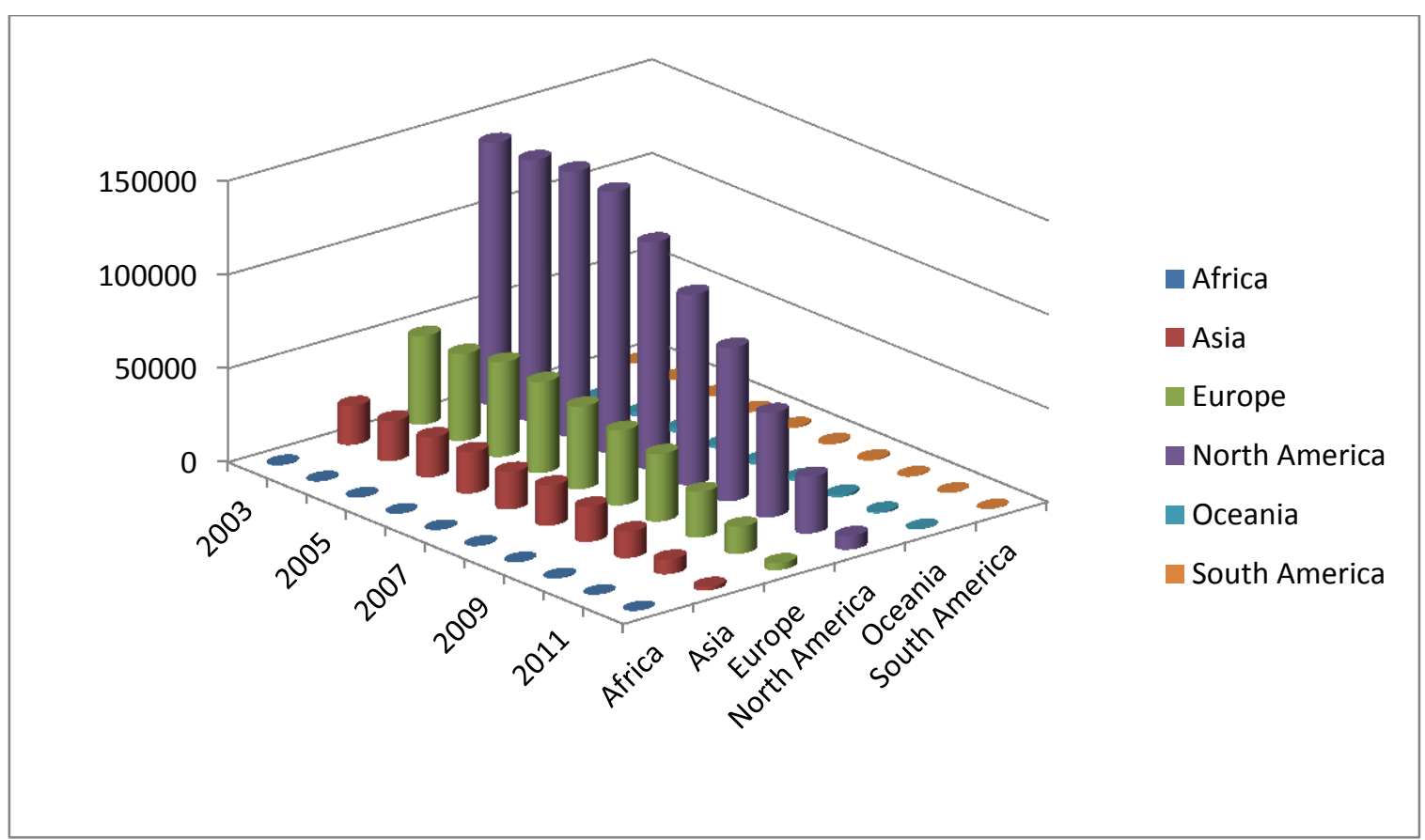

Figure 4. Self citation curves of oncology publications at continental level.

Self citation is a trend whereby a researcher cites his/her own previous work in any own new relevant work. The phenomenon is not new, and of the late the trend seems to more prevalent among researchers as the increased number of self citations refers a peer or reviewer to some other relevant works of the researcher carried out earlier. Self citation also increases the citation index of author hence betters his/her h-index scale, but this generally is not the intention behind self citing a document by an author. Of the total citation 5249511 received by oncology publication at global level during the period of study of them (1502900, $28.62 \%$ ) are self cited, which also means more than one-fourth of total citations.

Of the total self citation received at global level, North America leads the table with a share percentage of $(955458,63.57 \%)$, followed by Europe \& Asia with a share percentage of 
$(354567,23.59 \%) \&(169250,11.26 \%)$. Africa and South America have less than 1\% self citations, while as Oceania has $(16933,1.12 \%)$ self citations.

The self citations share percentage of the continents of the total citation received by publications at respective continental level, North America leads the table with a self citations share of $45.12 \%$, followed by Europe and Asia with their respective self citations share percentage of $16.02 \%$ \& $23.07 \%$.

Africa has a self citation share percentage of 9.25\%, Oceania $13.60 \%$, South America $11.61 \%$. Average self citations received by each oncology publication from each continent deserve to be analysed. North America is the leading continent which has maximum number of average 9.28 self citations for each oncology publication, followed by Europe and Asia with 2.84 and 2.39 average self citations respectively. Oceania has 2.31, South America 1.59 and Africa 0.85 average self citations in their each oncology research publication, published during the period of study.

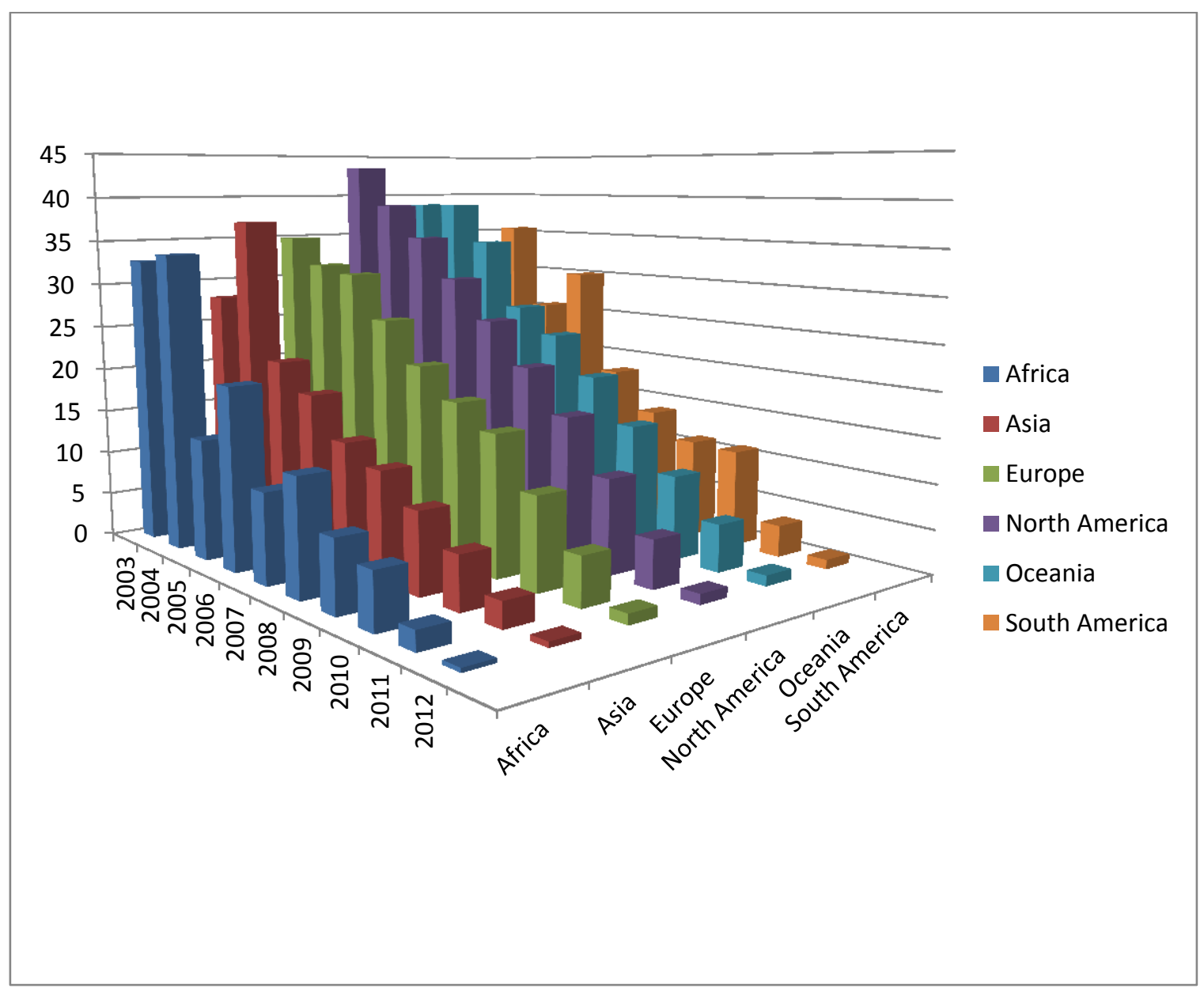

Figure 5. Frequency distribution of average annual citations at continental level. 
Table V. Yearly Average Citations Distribution of Publications at Continental Level.

\begin{tabular}{|c|c|c|c|c|c|c|c|}
\hline $\begin{array}{c}\text { Continent } \\
\text { Year } \downarrow\end{array}$ & Africa & Asia & Europe & N. America & Oceania & S. America & World \\
\hline $\mathbf{2 0 0 3}$ & 32.71 & 27.97 & 34.82 & 43.47 & 38.71 & 30.09 & 36.73 \\
\hline $\mathbf{2 0 0 4}$ & 33.73 & 27.13 & 31.79 & 38.82 & 38.79 & 35.60 & 33.79 \\
\hline $\mathbf{2 0 0 5}$ & 13.63 & 21.49 & 31.03 & 35.02 & 34.21 & 25.72 & 30.72 \\
\hline $\mathbf{2 0 0 6}$ & 20.44 & 18.49 & 26.12 & 30.38 & 26.47 & 30.07 & 26.19 \\
\hline $\mathbf{2 0 0 7}$ & 10.13 & 14.12 & 21.55 & 25.93 & 23.55 & 18.24 & 21.49 \\
\hline $\mathbf{2 0 0 8}$ & 13.00 & 12.16 & 18.30 & 21.20 & 19.19 & 13.92 & 17.81 \\
\hline $\mathbf{2 0 0 9}$ & 8.01 & 9.10 & 15.82 & 16.45 & 14.24 & 11.22 & 14.26 \\
\hline $\mathbf{2 0 1 0}$ & 6.27 & 5.97 & 10.39 & 10.68 & 9.52 & 11.02 & 9.28 \\
\hline $\mathbf{2 0 1 1}$ & 2.16 & 2.84 & 5.48 & 5.40 & 5.34 & 3.56 & 4.70 \\
\hline $\mathbf{2 0 1 2}$ & 0.46 & 0.61 & 1.18 & 1.15 & 1.17 & 1.05 & 1.00 \\
\hline Average & 14.05 & 13.98 & 19.64 & 22.85 & 21.11 & 18.04 & 19.59 \\
\hline
\end{tabular}

On average at global level, each oncology publication during the period of study received 19.59 citations, while as at continental level North America leads the table with an average 22.85 citations per article, followed by Oceania and Europe with an average 21.11 \& 19.64 citations per publication respectively. South America has received average 18.04 citations, Africa 14.05 and Asia 13.98 citation per publication. Older the publication higher is the number of citations received and lesser the number of publications better are the chances of receiving higher average citations.

Table VI. H-Index of Continents for Oncology Publications.

\begin{tabular}{|c|c|c|c|c|c|c|c|c|}
\hline $\begin{array}{c}\text { Continent } \rightarrow \\
\text { Year } \downarrow\end{array}$ & Africa & Asia & Europe & N. America & Oceania & S. America & $\begin{array}{c}\text { World } \\
\text { (Share\%) }\end{array}$ & CG\% \\
\hline $\mathbf{2 0 0 3}$ & 249 & 1193 & 3053 & 771 & 220 & 264 & $5750(9.80)$ & - \\
\hline $\mathbf{2 0 0 4}$ & 275 & 1211 & 3078 & 798 & 218 & 261 & $5841(9.95)$ & 1.58 \\
\hline $\mathbf{2 0 0 5}$ & 248 & 1216 & 3038 & 779 & 220 & 261 & $5762(9.82)$ & -1.35 \\
\hline $\mathbf{2 0 0 6}$ & 283 & 1231 & 3089 & 780 & 218 & 274 & $5875(10.01)$ & 1.96 \\
\hline $\mathbf{2 0 0 7}$ & 262 & 1206 & 3091 & 788 & 226 & 268 & $5841(9.95)$ & -0.57 \\
\hline $\mathbf{2 0 0 8}$ & 303 & 1242 & 3070 & 775 & 225 & 271 & $5886(10.03)$ & 0.77 \\
\hline $\mathbf{2 0 0 9}$ & 296 & 1245 & 3081 & 810 & 224 & 273 & $5929(10.10)$ & 0.73 \\
\hline $\mathbf{2 0 1 0}$ & 305 & 1234 & 3088 & 793 & 228 & 273 & $5921(10.09)$ & -0.13 \\
\hline $\mathbf{2 0 1 1}$ & 291 & 1245 & 3088 & 807 & 224 & 278 & $5933(10.11)$ & 0.20 \\
\hline $\mathbf{2 0 1 2}$ & 295 & 1252 & 3082 & 810 & 223 & 268 & $5930(10.10)$ & -0.05 \\
\hline $\begin{array}{c}\text { Average } \\
\text { (Share\%) }\end{array}$ & 280.70 & 1227.50 & 3075.80 & 791.10 & 222.60 & 269.10 & 5866.80 & 3.14 \\
$(4.78)$ & $(20.92)$ & $(52.42)$ & $(13.48)$ & $(3.79)$ & $(4.58)$ & $(100)$ & $(0.31)$ \\
\hline
\end{tabular}

Hirsch or h-index as known to all of the latter has become one of the major parameter to measure and quantify the scientific output in terms of quality. h-index is no more confined to scientific publications as such has moved beyond it and today we can see h-index has become a quality measuring technique for every kind of literature, of individuals, of groups, of journals, of countries, of continents and of course last but not least of the world as whole. Given the fact, h-index of the oncology publications at global level during the period of study, on average remained at 5866.80 with a continental share percentage in Europe (3075.80, $52.42 \%)$, Asia $(1227.50,20.92 \%)$, North America (791.10, $13.48 \%$ ), Africa $(280.70,4.78 \%$ ), South America (269.10, $4.58 \%) \&(222.60,3.79 \%)$. 
On average the h-index of oncology publications at global level grew at $0.31 \%$ annually during the period of study.

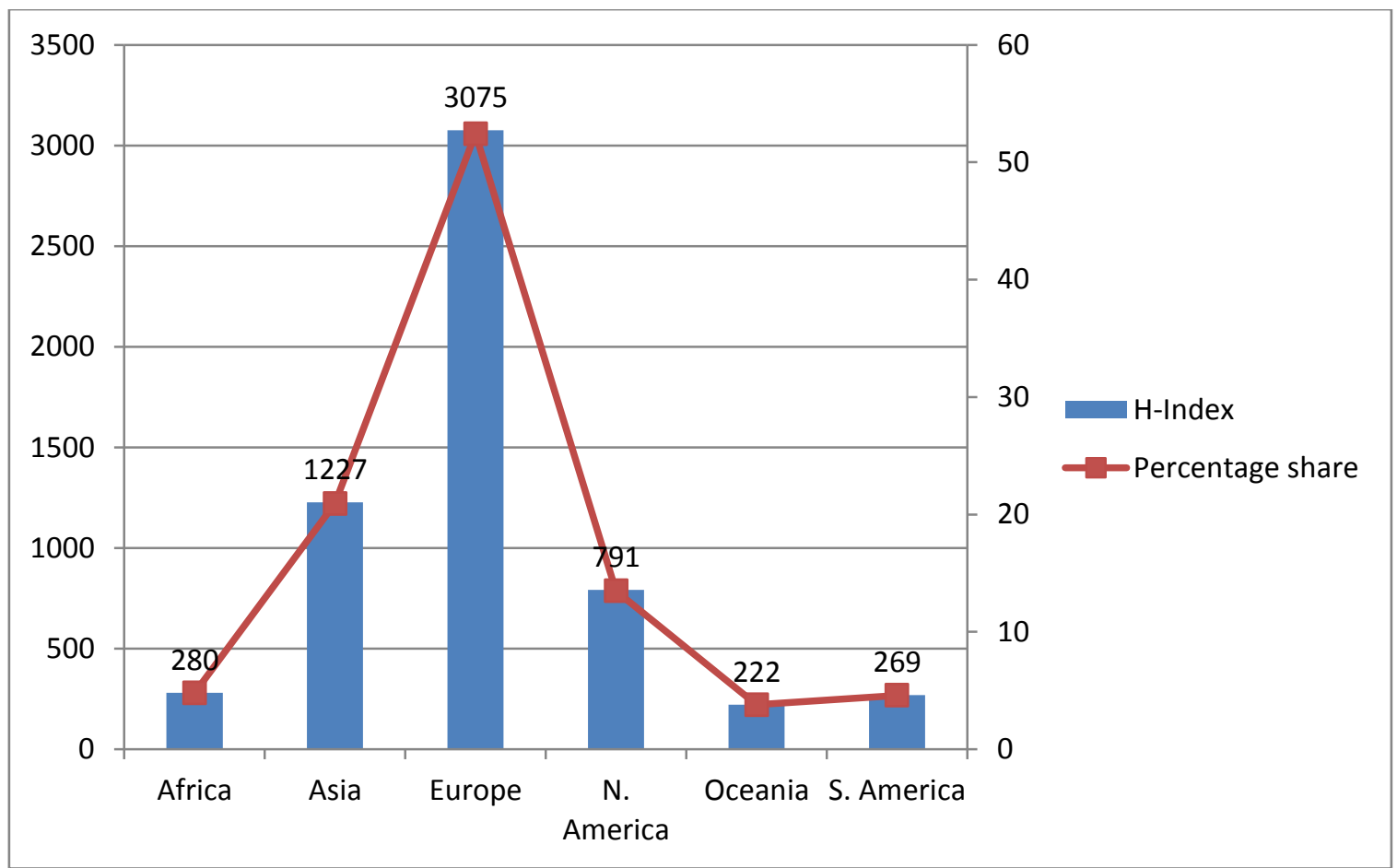

Figure 6. H-Index of Continents for Oncology Publications.

\section{CONCLUSIONS}

Africa, Oceania \& South America compared to their counterparts like, Asia, Europe and North America are the continents which have made very little contribution to global oncology research. Africa and South America are the continents which mostly comprises of the lesser developed nations, hence cannot press sufficient money towards research activities in the given field. Agencies like WHO, World Bank, IMF, etc. has got to play a very vital role whereby they can lend helping hand to all under developing countries to create the infrastructure so as to undertake research in the given field. Developed nations can extend financial support and can also train their human resource to explore the hitherto untouched biomedical research areas.

Europe and North America are the two leading continents of the world which have significantly contributed to the global oncology research. Countries like the United States, Italy, France, Germany, United Kingdom, Japan and China are some of the leading oncology research countries of the world and rightly so for the fact that these are the most developed nations of the world. At continental level US dominates the scene in North America, Japan and China in Asia and the rest in Europe.

There are also some smaller and under developing nations which coexist with developed ones in developed regions of the world, but for the want of adequate facility and resources are not able to contribute in the given field. These smaller nations are mostly dependent on the developed nations for the advanced medical attention and other such 
healthcare facilities which they are unable to raise themselves in their own country. There is always greater need that research and developmental activities in the field of biomedical should at least reach to the sustainable level and this can be achieved by encouraging collaborative research among different research institutions, organizations, countries, or even for that matter Individuals can contribute their own way by sharing their expertise and knowledge about oncology research among those who are working in this sphere.

\section{References}

[1] International Agency for Research on Cancer. (2014). World Cancer Report 2014. World Health Organization: Geneva.

[2] Ibid.

[3] Ibid.

[4] Ferlay J., Shin H. R., Bray F., et al. (2010). GLOBOCAN, 2008 v2. 0, Cancer Incidence and Mortality Worldwide: IARC Cancer Base No. 10 [Internet]. Lyon: IARC. Available at: http://globocan.iarc.fr.

[5] International Agency for Research on Cancer. (2014). World Cancer Report 2014. World Health Organization: Geneva.

[6] Micheli A., Di Salvo, F., Lombardo C., Ugolini D., Baili P., Pierotti M., Tumori 97(6) (2011) 683-689.

[7] Grossi F., Belvedere O., Rosso R., European Journal of Cancer 39(1) (2003) 106-111.

[8] Hortobagyi G. N., de la Garza Salazar J., et al., Clinical breast cancer 6(5) (2005) 391-401.

[9] Glynn R. W., Scutaru C., Kerin M. J., Sweeney K. J., Breast Cancer Res. 12(6) (2010) R108.

[10] Ugolini D., Mela G. S., European Journal of Cancer 39(13) (2003) 1888-1894.

[11] Glynn R. W., Chin J. Z., Kerin M. J., Sweeney K. J., PLoS One 5(11) (2010) e13902.

[12] Ortiz A. P., Calo W. A., Suárez-Balseiro C., Maura-Sardo M., Suárez E., Revista Panamericana de Salud Pública 25(4) (2009) 353-361.

[13] Pandita, R., Singh, Shivendra., \& Ramesh, C. (2014). Research Output of some Selected Indian Medical Research Institutions (2007-2011). Library Philosophy and Practice (e-Journal). Paper 1065. http://digitalcommons.unl.edu/libphilprac/1065 